\title{
NUCLEOTIDE SEQUENCE OF THE GENE FOR THREONINE DEAMINASE (ILV1) OF SACCHAROMYCES CEREVISIAE
}

by

\author{
MORTEN C. KIELLAND-BRANDT, STEEN HOLMBERG, \\ JENS G. LITSKE PETERSEN
}

and

TORSTEN NILSSON-TILLGREN

\author{
Department of Physiology, Carlsberg Laboratory, \\ Gamle Carlsberg Vej 10, DK-2500 Copenhagen Valby \\ and \\ 'Institute of Genetics, University of Copenhagen \\ Oster Farimagsgade 2A, DK-1353 Copenhagen K
}

\begin{abstract}
Keywords: Isoleucine-valine biosynthesis, allosteric enzyme, upstream open reading frame, general amino acid control, multivalent repression
\end{abstract}

The 1,728 base pair coding region of the $I L V I$ gene and the adjacent 777 base pair upstream and 261 base pair downstream regions were sequenced. The size of the polypeptide deduced from the coding region is larger than previously determined values for the molecular weight of threonine deaminase, suggesting that a peptide with a molecular weight of about 15,000 is cleaved off in connection with the intracellular processing of the enzyme.

In front of the gene are found three copies of the element TGACT characteristic for genes under general amino acid control. Two of the copies are present in an onientation which is reversed to that found in front of other genes. Sites which potentially can bind strongly to the 3 '-end of yeast $18 \mathrm{~S}$ ribosomal RNA are present at positions -5 and -24 from the start of the coding region.

An open reading frame of 31 codons is found in front of the gene. Its translation initiator codon is located at the center of a $15 !$ base pair imperfect tandem inverted repeat which in a single-stranded nucleic acid would fold into a stem structure with a $\Delta G$ of about $-33 \mathrm{kcal} \cdot \mathrm{mole}^{-1}$. The biological significance of this arrangement is unknown.

\section{INTRODUCTION}

The biosynthesis of isoleucine starts with the conversion of threonine to $\alpha$-ketobutyrate and ammonia, a step catalysed by threonine deaminase (threonine dehydratase, 4.2.1.16). The enzyme in yeast has been purified $(1,14)$. It is a tetramer with a molecular weight of $4 \times 48,000$ (1), and complexed in mitochondria with other enzymes involved in the biosynthesis of isoleu- cine and valine (24). It contains pyridoxal phosphate as a non-covalently bound prosthetic group. The catalytic activity is subject to (feed back) inhibition by isoleucine and is dependent in a complicated manner on the concentrations of isoleucine, threonine and valine (1). Determination of the amino acid sequence of the apoenzyme should ultimately be of help in understanding these interactions.

Abbreviations: $\mathrm{bp}=$ base pairs; $\mathrm{kb}=$ kilo bases; $\mathrm{kD}=$ kiloDalton 
The amount of threonine deaminase in the cell is regulated. The simultaneous presence of isoleucine, valine and leucine in the growth medium causes repression (so-called multivalent repression) of the synthesis of the enzyme (5). Furthermore, its synthesis is subject to general amino acid control (cf. 29). The gene, $I L V I$, for the apoenzyme, is nuclear and located on chromosome $\mathrm{V}$. It has recently been isolated on a bacterial plasmid and its approximate position on the restriction endonuclease map was determined by DNA-RNA hybridization and confirmed genetically (21). Sequencing of the gene and its adjacent regions was considered important, not only to obtain the amino acid sequence, but also as a the first step to identify DNA regions involved in multivalent repression. A minor part of the sequence determined in the present work has been previously reported (29).

\section{MATERIALS AND METHODS}

\subsection{Preparative electrophoresis}

Specific DNA fragments in restriction endonuclease digests were selected by preparative agarose gel electrophoresis using DE81 ion exchange paper (8) or dialysis membrane (30).

\subsection{DNA sequencing by the dideoxy method}

Standard procedures using the singlestranded bacteriophage M13 vectors (19) and dideoxynucleotide sequencing were employed (25). Thus, restriction fragments were ligated into the replicative form of $\mathrm{M} 13 \mathrm{mp8}$ or M13mp9 (19) and Escherichia coli strain JM 101 (18) was transfected with the mixture and plated for development of plaques. For two EcoRI fragments the principles for systematic sequencing described by HONG (13) were followed.

Electrophoresis, handling of gels and sources of vectors, bacterium, chemicals and materials were as described $(4,23)$. Autoradiography was carried out at room temperature for 1-5 days without intensifying screens.

The nucleotide sequence data handling was carried out on a Honeywell DPS-8. The programs were kindly supplied by Dr. R. STADEN, MRC, Cambridge, England.

\section{RESULTS}

Plasmid pC516 contains the functional $I L V I$ gene (21). Figure 1 shows the relevant part of the plasmid. It was treated with PstI and fragments of different sizes were prepared on agarose gels. After further treatment of the isolated fragments
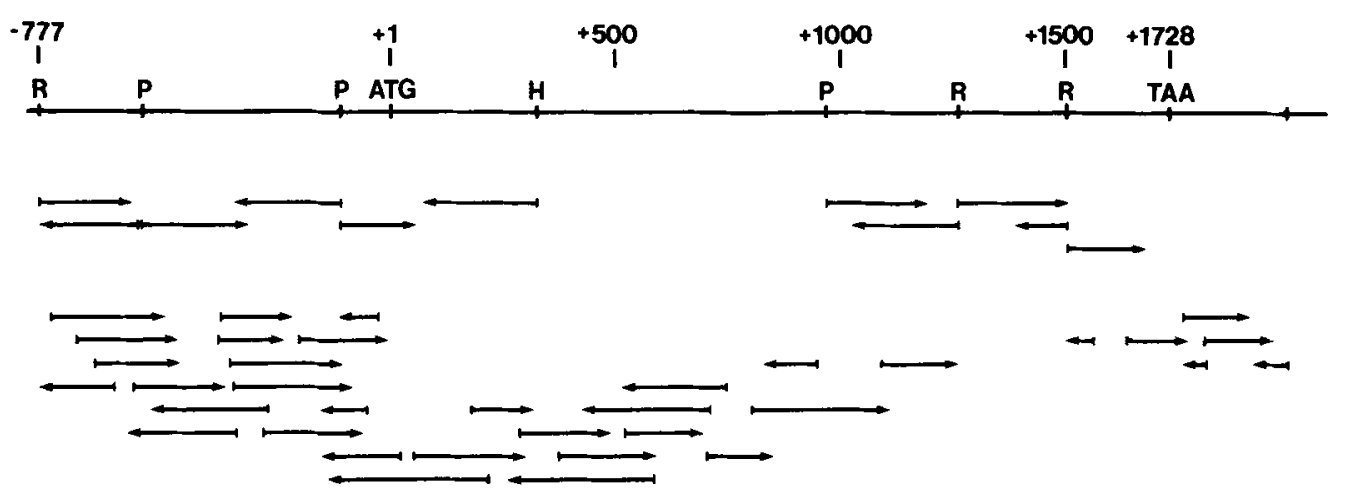

Figure 1. Map of the nucleotide sequence readings from individual recombinant M13 clones used to establish the sequence of the $[L \mathrm{VJ}$ gene. (A) designate clones obtained from restriction endonuclease fragments using EcoRI (R), HindIII (H) or PstI (P). The two sets of ctones designated (B) were obtained from a 2,035 bp EcoRI fragment (left) and a $1.6 \mathrm{~kb}$ EcoRI fragment (right) using DNase I to generate random start-points for sequencing. The 3 clones $(\mathrm{C})$ were constructed from BglII-Haell fragments in order to establish the sequences across the two EcoRI sites. 
with EcoRI, Sall, HindIII or combinations thereof, subfragments were prepared. The primary Pstl fragments and the subfragments were inserted in $\mathrm{M} 13$ vectors and sequenced. This series of experiments yielded sequences indicated by the group of arrows designated $A$ in Figure 1.

The sequences corresponding to arrows $\mathrm{B}$ were obtained from two EcoRi fragments (2,035 bp and $1.6 \mathrm{~kb})$ of pC516 by the DNase I method of HONG (13). To establish sequences overlapping the two EcoRI sites between the two groups of arrows (B), sequencing from a BgllI site at $+1,246$ and an Haell site at $+1,540$ was performed after cloning of the appropiate fragments into M13mp8 (arrows C). Approximately $80 \%$ of the sequence was obtained from both strands.

Figure 2 shows the sequence spanning an EcoRI site located 777 bases upstream from the ATG of a 1,728 bp long open reading frame and a point $2 \overline{6} \mathrm{I}$ bases downstream of the last codon.

\section{DISCUSSION}

The open reading frame of 1,728 bases is the coding region for the $I L V I$ gene since it covers the stretch which by gene splitting has been shown to be essential for the $I L V I$ function (21). In addition, an mRNA of 2,000 nucleotides is transcribed from this region (21). It is the only reading frame in this neigbourhood that can accommodate a protein of $48 \mathrm{kD}$, the size found for the monomer of threonine deaminase $(1,14)$. We have considered the possibility that alternative, smaller reading frames assembled with one or more introns could constitute the $I L V I$ coding region, but found this highly unlikely. First, introns are rare in nuclear genes of Saccharomyces and they have not been found in amino acid biosynthetic genes. Secondly, an intron would expectedly contain the sequence TACTAAC (22) and this sequence does not occur here.

The $I L V I$ gene can encode a polypeptide with a molecular weight of 63,719 while the monomer of threonine deaminase has been determined to be only about $48 \mathrm{kD}$. To explain this difference one could imagine that the mRNA starts within the open reading frame and that the methionine codon at +346 or +466 were used as the start of translation. However, mRNA characterization (to be published) has shown

Table I. Codon usage in the yeast $I L V I$ gene

\begin{tabular}{|c|c|c|c|c|c|c|c|c|c|c|c|}
\hline \multicolumn{12}{|c|}{ Second letter } \\
\hline & & \multicolumn{2}{|c|}{$\mathrm{T}$} & \multicolumn{2}{|c|}{$\mathrm{C}$} & \multicolumn{2}{|l|}{ A } & \multicolumn{2}{|c|}{$\mathrm{G}$} & & \\
\hline & & Phe & 10 & Ser & 21 & Tyr & 10 & Cys & 5 & $\mathrm{~T}$ & \\
\hline & & Phe & 13 & Ser & 9 & Tyr & 10 & Cys & 0 & $\mathrm{C}$ & \\
\hline & $T$ & Leu & 15 & Ser & 5 & ochre & 0 & opal & 0 & A & \\
\hline & & Leu & 21 & Ser & 1 & amber & 0 & Trp & 1 & G & \\
\hline$F$ & & & & & & & & & & & $\mathrm{~T}$ \\
\hline$i$ & & Leu & 8 & Pro & 15 & His & 12 & Arg & 9 & $T$ & $\mathrm{~h}$ \\
\hline$r$ & & Leu & 0 & Pro & 4 & His & 6 & Arg & 1 & $\mathrm{C}$ & $\mathrm{i}$ \\
\hline $\mathbf{s}$ & C & Leu & 9 & Pro & 8 & Gln & 19 & Arg & 0 & A & $r$ \\
\hline$t$ & & Leu & 2 & Pro & 0 & Gln & 4 & Arg & 1 & G & $d$ \\
\hline 1 & & Ile & 17 & Thr & 20 & Asn & 10 & Ser & 2 & $\mathrm{~T}$ & 1 \\
\hline $\mathrm{e}$ & & Ile & 14 & Thr & 5 & Asn & 16 & Ser & 2 & C & $\mathrm{e}$ \\
\hline$t$ & A & Ile & 2 & Thr & 2 & Lys & 17 & Arg & 17 & A & $\mathrm{t}$ \\
\hline $\mathrm{t}$ & & Met & 10 & $\mathrm{Thr}$ & 4 & Lys & 17 & Arg & 4 & G & $t$ \\
\hline e & & & & & & & & & & & $\mathrm{e}$ \\
\hline \multirow[t]{4}{*}{$r$} & & Val & 26 & Ala & 24 & Asp & 21 & Gly & 32 & $\mathrm{~T}$ & $r$ \\
\hline & & Val & 13 & Ala & 14 & Asp & 6 & Gly & 4 & C & \\
\hline & $G$ & Val & 8 & $\mathrm{Ala}$ & 1 & Glu & 30 & Gly & 3 & A & \\
\hline & & Val & 8 & Ala & 2 & Glu & 4 & Gly & 2 & G & \\
\hline
\end{tabular}


GAATTCG TTCTCATTAC ATGGCCGCCC ACCATGCACC TTTACATTTC GTATTTCTTT $-700$

CCTCTCTGCG CTATATCTMT GGGAAAGATT TGCAATCAAG GCAAACACCC GCTTGTCCCC TTTCAATTCT TGTGTTATTT $-600$

CAAAAACCAT CCTTTCATAA TATATWTGTA TPTCITTCGC CCTGGACTCT ACCAGTTCAT ATTTAGAACC TTCCACATAT $-500$

CGTCCAATTC TGCAGCCCAC ATCTTTTTCC ACCACGATAC GGGAAACAGA ATGGGTCCTT GGATTCTCGC TAAACAGTCT $-400$

CTCTATTCCC CTGTTCAAAA CCCTCAAGAT ATTTGTTTCC CGCAACAGCT GCAATTGCAA TTGATCAATC CTATGCGAAA

ATGCCGAGTT TATGTTATTC AagACGCATT TTAAAAAATT CACTAGCGGC TCCTTGAAAT TCATTATgTC TGATGAataT $-300$

GAAAACCTTT TCCT⿱GACTAC CAAGACTCTT TAACTCTTCT CTCTTTATTG CATATTATCT CTGCTATTTT GTGACGTTCA $-200$

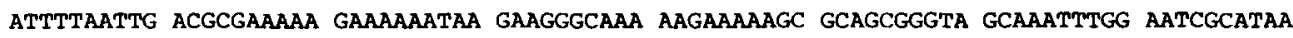
$-100$

AAAGAAAAAA AAAATATCAA AGAAAAAGAG TCATCTCAAA CATATGTCTG CAGATACTTC ATTATCAGCT TTGAAAACTT

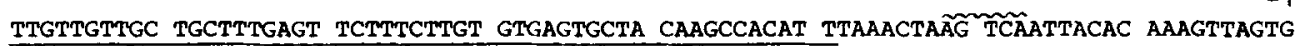
stp

$+1$ 50

ATG TCA GCT ACT CTA CTA AAG CAA CCA TTA TGT ACG GTT GTT CGG CAA GGT AAA CAG TCC AAA GTG met ser ala thr leu leu lys gln pro leu cys thr val val arg gln gly lys gln ser lys val 100

TCT GGA TTG AAC CTT TTG AGA CTA AAG GCT CAT TTG CAC AGA CAA CAC CTG TCA CCT TCC TTG ATA ser gly leu asn leu leu arg leu lys ala his leu his arg gln his leu ser pro ser leu ile 150

AAA CTA CAC TCT GAA TTG AAA TTG GAT GAG CTG CAA ACT GAT AAC ACC CCT GAT TAC GTC CGT TTA lys leu his ser glu leu lys leu asp glu leu gln thr asp asn thr pro asp tyr val arg leu 200 250

GTT TTA AGG TCC TCT GTA TAC GAT GTT ATT AAT GAA TCT CCA ATC TCT CAA GGT GTA GGT TTG TCT val leu arg ser ser val tyr asp val ile asn glu ser pro ile ser gln gly val gly leu ser 300

TCC CGT CTA AAC ACG AAT GTC ATC TTG AAA AGA GAA GAT CTA TTG CCT GTT TTC TCT THC AAG CTM ser arg leu asn thr asn val ile leu lys arg glu asp leu leu pro val phe ser phe lys leu 350

CGT GGT GCC TAT AAC ATG ATT GCC AAG TTG GAC GAT TCT CAA AGA AAC CAG GGT GTT ATT GCC TGT arg gly ala tyx asn met ile ala lys leu asp asp ser gln argasn gln gly val ile ala cys 400 450

TCA GCT GGG AAT CAT GCC CAA GGT GTG GCC TTT GCT GCT AAA CAC TTG AAA ATA CCT GCT ACT ATC ser ala gly asn his ala gln gly val ala phe ala ala lys his leu lys ile pro ala thr ile 500

GTT ATG CCT GTT TGT ACA CCA TCT ATT AAG TAT CAA AAT GTC TCG AGA TTA GGG TCT CAA GTC GTC val met pro val cys thr pro ser ile lys tyr gln asn val ser arg leu gly ser gln val val 550

CTA TAT GGT AAC GAT TTT GAC GAG GCT AAG GCT GAA TGT GCC AAA TTG GCT GAA GAG CGT GGC TTG leu tyr gly asn asp phe asp glu ala lys ala glu cys ala lys leu ala glu glu arg gly leu 600 650

ACG AAC ATT CCT CCT TTC GAT CAT CCT TAT GTC ATT GCC GGT CAA GGT ACT GTA GCT ATG GAA ATC thr asn ile pro pro phe asp his pro tyr val ile ala gly gln gly thr val ala met glu ile 700

CTA AGA CAA GTA CGT ACC GCT AAT AAG ATC GGT GCT GTC TTT GTT CCC GTC GGC GGT GGT GGT TTA leu arg gln val arg thr ala asn lys ile gly ala val phe val pro val gly gly gly gly leu

Figure 2. DNA sequence of the gene for threonine deaminase (ILVI) of Saccharomyces cerevisiae. The A of the translation initiation codon is numbered +1 . The preceding $G$ is numbered -1 . The amino acid sequence of the primary transiation product is given as inferred from the nucleotide sequence. Several sequence elements of possible significance can be recognized outside the coding region: The sequence TGACT and its complement AGTCA, implicated in general amino acid control, are marked with wavy lines. TATA-like sequences are designated with dotted lines. At position -117 starts a reading frame of 31 codons which is underlined. Its ATG and the preceding CAT provide the symmetry point of a large imperfect inverted repeat. The sequences TATGT and TATTTATA signify possible control elements for termination of transcription and are marked with broken lines. 
ATT GCT GGT ATT GGT GCT TAT TTG AAA AGG GTT GCT CCT CAT ATC AAA ACT ATP GGT GTS GAA ile ala gly ile gly ala tyr leu lys arg val ala pro his ile lys thr ile gly val glu 800

850

ACT TAC GAT GCG GCC ACT TTA CAT AAT TCC TTG CAA CGC AAC CAG AGA ACT CCT TTA CCT GTG thr tyr asp ala ala thr leu his asn ser leu gln arg asn gln arg thr pro leu pro val 900

GTG GGT ACT TTT GCC GAT GGT ACG TCT GTG CGT ATG ATT GGT GAA GAA ACA TTT AGA GTC GCC val gly thr phe ala asp gly thr ser val arg met ile gly glu glu thr phe arg val ala 950

CAA CAA GTG GTP GAT GAA GTT GTT CTT GTT AAC ACT GAC GAA ATC TGT GCT GCA GTA AAG GAT gln gln val val asp glu val val leu val asn thr asp glu ile cys ala ala val lys asp 1000

ATT TTT GAA GAT ACT AGA AGT ATT GTA GAA CCA TCT GGT GCC CTT TCA GTA GCC GGT ATG AAG ile phe glu asp thr arg ser ile val glu pro ser gly ala leu ser val ala gly met lys 1050 1100

AAA TAC ATC TCT ACC GTA CAT CCA GAA ATT GAC CAC ACT AAA AAC ACC TAT GTT CCC ATC CTT lys tyr ile ser thr val his pro glu ile asp his thr lys asn thr tyr val pro ile leu 1150

TCT GGT GCT AAC ATG AAC TTT GAT AGA TTA AGA TTT GTT TCC GAA CGP GCT GTP CTP GGT GAA ser gly ala asn met asn phe asp arg leu arg phe val ser glu arg ala val leu gly glu 1200

GGA AAG GAA GTC TTC ATG TTA GTT ACT TTA CCC GAC GTC CCT GGT GCG TTC AAG AAA ATG CAA gly lys glu val phe met leu val thr leu pro asp val pro gly ala phe lys lys met gln 1250

AAG ATC ATC CAC CCA AGA TCT GTC ACT GAA TTC TCT TAC CGT TAC AAT GAA CAT CGT CAT GAG lys ile ile his pro arg ser val thr glu phe ser tyr arg tyr asn glu his arg his glu 1300 1350

TCC TCT AGT GAA GTG CCC AAG GCT TAC ATT TAC ACT TCT TTC AGC GTC GTT GAC AGA GAA AAG ser ser ser glu val pro lys ala tyr ile tyr thr ser phe ser val val asp arg glu lys 1400

GAA ATC AAG CAA GTT ATG CAA CAG TTG AAT GCT TTA GGT TTT GAA GCT GTG GAT ATC TCC GAT glu ile lys gln val met gln gln leu asn ala leu gly phe glu ala val asp ile ser asp 1450

AAC GAA TTG GCT AAA TCT CAT GGT AGA TAC TTG GTT GGT GGT GCT TCT AAG GTT CCT AAT GAA asn glu leu ala lys ser his gly arg tyr leu val gly gly ala ser lys val pro asn glu 1500

AGA ATT ATT TCA TTT GAA TTC CCT GAA AGA CCA GGT GCC TTG ACT AGG TTC CTT GGA GGC CIA arg ile ile ser phe glu phe pro glu arg pro gly ala leu thr arg phe leu gly gly leu 1550 1600

AGC GAT TCT TGG AAT CTT ACT TTA THC CAT TAT AGA AAC CAT GGT GCC GAT ATC GGT AAG GTT ser asp ser trp asn leu thr leu phe his tyr argasn his gly ala asp ile gly lys val 1650

TTA GCT GGT ATT TCC GTT CCT CCA AGG GAA AAC TTA ACC TTC CAA AAA TTC TTG GAA GAT TTA leu ala gly ile ser val pro pro arg glu asn leu thr phe gln lys phe leu glu asp leu 1700

1728

GGC TAC ACT TAT CAT GAT GAA ACT GAT AAC ACT GTT TAT CAA AAA TIC TTG AAA TAT TAA AAC gly tyr thr tyr his asp glu thr asp asn thr val tyr gln lys phe leu lys tyr stp 1800

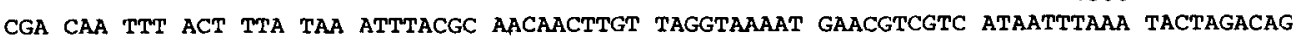
$\operatorname{stp}$

CGCGTGTATG TTACTCATAT TTATACTCAT ATCATGTGA CCTATAAACC AATCAATAC AAGATTTACA GTTCTCTTGT 1900

TTTCTTCTTC CAGTTTCCC CTTTTATTTC CTGRAGAATA AACTTAAACA GTGATCATTA ATMTATAATT TTCTGCTTTG 1989

TAAATACGGC GGTAATGGA 
that all transcription in this region starts upstream of the ATG at position +1. It is therefore suggested that the primary translation product has a size of $64 \mathrm{kD}$ and is processed by proteolytic cleavage into the $48 \mathrm{kD}$ monomer of threonine deaminase. In fact, such processing is known to occur for many proteins that are synthesized in the cytoplasm and transported into the mitochondria (cf. 20). Determination of the amino acid sequence of the N-terminal region of the mature protein will allow positioning of the cleavage point.

Regarding the codons of the $I L V I$ gene, we have noted that the tryptophan codon is only found once. Another feature is that the first part of the gene is very rich in leucine codons, 14 out of the first 55 codons, i.e. $25 \%$ are leucine codons. This is not associated with any particular hydrophobicity of this region as judged by an analysis of the hydrophobicity along the polypeptide chain according to the method of KYTE and DoOlitTle (15). This leucine rich part of the translation product is suggested (see discussion above) not to be present in the mature threonine deaminase. Codon usage (Table I) has a significant, but relaxed bias towards the codons commonly used in yeast (3), which might be expected for a protein present in moderate amounts in the cell (3). According to the enzyme activity of crude cell extracts as compared to purified enzyme $(1,14)$, threonine deaminase makes up about $0.1 \%$ of total cell protein.

Upstream to the coding region several sequence elements of interest can be recognized. DONAHUE et al. (6) and HINNEBUSCH and FINK (12) have suggested that the element TGACT plays an important role for general amino acid control. This element is found three times in front of the $I L V I$ coding region, once in the orientation in which it has been identified in the 5 ' non-coding regions of HIS4, HISI, HIS 3 and TRP5 (cf. 12) and twice in reverse orientation, i.e. AGTCA in the strand presented in Figure 2. The element TGACT is the most conserved core of the larger consensus sequence $\mathrm{A}(\mathrm{A} / \mathrm{T}) \mathrm{GTGACTC}(12)$. The sequence element in front of $I L V I$ that comes closest to this consensus is AGATGACTC which is the complement to the 9 base pair sequence starting at -133 in Figure 2. Determination of threonine deaminase activity after the addition of 5methyltryptophan to the growth medium (cf. 29) has shown that $I L V I$ is under general control. In vitro mutagenesis will permit us to determine the significance of the TGACT containing elements in front of $I L V I$.

The element TATAAA or similar sequences are common in front of yeast genes. They are believed to have promoter potential (cf. 9) and at least in some cases $(10,27)$, in vitro mutations give phenotypes that confirm this notion. In front of $I L V I$ there are two similar elements, TATGAAA at -323 and TATCAAA at -146 , the latter being at a distance from the coding region comparable to that of other yeast genes (cf. 26).

We have noticed two more features in the front region of $I L V I$. When the upstream region is searched for heptanucleotides complementary to the 3 '-end of yeast $18 \mathrm{~S}$ ribosomal RNA, three segments at positions $-177,-24$ and -5 are found. With the presumed TATA-box at position -146 , only the latter two possible ribosome binding sites should be considered. Using energy calculations for intramolecular base pairing according to TiNoco et al. (28) to compare bimolecular binding energies, the sequence TAAGTC (at position -24 ) yields a relative $\Delta G$ of -3.6 $\mathrm{kcal} \cdot \mathrm{mole}^{-1}$ when binding to the 3'-end of $18 \mathrm{~S}$ RNA and the relative $\Delta \mathrm{G}$ is $-8 \mathrm{kcal} \cdot \mathrm{mole}^{-1}$ for the sequence TAGTGAT (at position -5 ). Sequences complementary to $18 \mathrm{~S}$ ribosomal RNA of five mRNA species are discussed by ZALKIN and YANOFSKY (31). The sequence of the ILVI mRNA at -24 can bind to the $18 \mathrm{~S}$ rRNA with a strength comparable to that of these five mRNA's while the sequence at position -5 binds more strongly. In comparison to other yeast mRNA's the two putative ribosome binding sites at $I L V I$ are positioned very close to the ATG. These facts make it attractive to investigate the level of gene expression in strains with site-directed mutagenesis in this region.

The other feature of the $I L V I$ front region that we have noted is the following. At position -117 is the ATG of an open reading frame that could potentially code for a polypeptide of 31 amino acids. Upstream open reading frames are not uncommon to amino acid biosynthetic genes in yeast $(2,7,12,31)$. In the case of $L E U 2$, the upstream reading frame is rich in leucine codons 
and is associated with potential foldback structures, facts that have been proposed to be of significance for the repression of the gene by leucine (2). Subsequent deletion analysis has shown that a small palindromic $\mathrm{G}+\mathrm{C}$-rich region, but not the reading frame, is important for regulation of $L E U 2$ (17). Similarly, in the case of HIS4 a deletion analysis has shown that the two open reading frames present in the 5 ' non-coding region are not required in either cis or trans for the regulation of HIS4 by the general control system (16). The ATG of the open reading frame

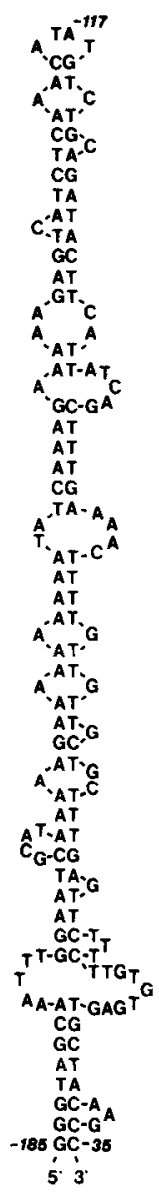

Figure 3. Hypothetical folding of single-stranded nucleic acid with the sequence present from -185 to -35 of the yeast $I L V I$ gene. Calculation according to TiNOCO et al. (28) for the corresponding RNA molecule gives a $\Delta \mathrm{G}$ of folding of $-33 \mathrm{kcal} \cdot \mathrm{mole}^{-1}$. At the top of the hairpin loop starts a reading frame potentially coding for a polypeptide of 31 amino acids. in front of of $I L V I$ and the preceding CAT provide the symmetry point of a large imperfect inverted repeat. In a computer search in the region from -777 to +330 , i.e. well into the coding region, for inverted repetitions of segments of 15 bases with an allowance of up to two imperfections, this palindrome was the most pronounced and regular one. A single-stranded nucleic acid of this sequence is expected to fold into the structure represented in Figure 3. It must be emphasized that this foldback structure is hypothetical but illustrates the extent of the inverted repetition.

At the 3' end of the gene the TAA stop codon at position $+1,729$ is followed by a second TAA in frame at position $+1,750$. Two sequences have been suggested to be important for termination of transcription in yeast and both are present downstream from the $I L V I$ coding region. The sequence TATGT seems to play a role in transcription termination of the $\mathrm{CYCl}$ gene (32). This sequence is found at position $+1,817$ of $I L V I$. At position $+1,828$ there is the sequence TATTTATA, differing at only one position from the sequence element TTTTTATA, suggested by HENIKOFF et al. (11) to be important for termination of the Drosophila $A D E 8$ message in yeast.

\section{ACKNOWLEDGEMENTS}

Of particular importance for this work has been the introduction at Department of Physiology of the M13 sequencing technique by cand. polyt. SøREN K. RASMUSSEN. We are very grateful for his maintenance of equipment and software, and his readiness to always help solve problems. The skilful technical assistance by Ms. GITTE BANK and the stimulating discussions with Professor Diter von Wettstein, Dr. Julio Polaina and cand. scient. ClaEs GJeRMANSEN are gratefully acknowledged. This work was supported by grant 16-3344.H656 from the Danish National Research Council for Technical Sciences and grant 133/001-84.154 from Teknologirådet of the Ministry of Industry to Professor Diter von WeTtSTEIN. 


\section{REFERENCES}

1. AHMEd, S.I., A.P. Bollon, S.J. Rogers \& P.T. MAGEE: Purification and properties of threonine deaminase from Saccharomyces cerevisiae. Biochimie 58, 225-232 (1976)

2. ANDREADIS, A., Y.-P. HsU, G.B. Kohlhaw \& P. SCHIMMEL: Nucleotide sequence of the yeast LEU2 shows 5'-noncoding region has sequences cognate to leucine. Cell 31, 319-325 (1982)

3. Bennetzen, J.L. \& B.D. Hall: Codon selection in yeast. J. Biol. Chem. 257, 3026-3031 (1982)

4. BigGin, M.D. T.J. Gibson \& G.F. Hong: Buffer gradient gels and ${ }^{33} \mathrm{~S}$ label as an aid to rapid DNA sequence determination. Proc. Nat. Acad. Sci. USA 80, 3963-3965 (1983)

5. BUSSEY, H. \& H.E. UMBARGER: Biosynthesis of branched-chain amino acids in yeast: Regulation of synthesis of the enzymes of isoleucine and valine biosynthesis. J. Bacteriol. 98, 623-628 (1969)

6. Donahue, T.F.. R.S. Daves, G. Lucchini \& G.R. FINK: A short nucleotide sequence required for regulation of $H I S 4$ by the general control system of yeast. Cell 32, 89-98 (1983)

7. Donahue. T.F., P.J. Farabaugh \& G.R. Fink: The nucleotide sequence of the HIS4 region of yeast. Gene 18, 47-59 (1982)

8. Dretzen, G., M. Bellard, P. Sassone-Corsi \& P. Chambon: A reliable method for the recovery of DNA fragments from agarose and acrylamide gels. Anal. Biochem. 112, 295-298 (1981)

9. Guarante. L.: Yeast promoters: Positive and negative elements. Cell 36, 799-800 (1984)

10. GuARANTE, L. \& T. Mason: Heme regulates transcription of the $\mathrm{CYCl}$ gene of S. cerevisiae via an upstream activation site. Cell 32, 1279-1286 (1983)

11. Henikoff, S. J.D. Kelly \& E.H. Cohen: Transcription terminates in yeast distal to a control sequence. Cell 33, 607-614 (1983)

12. HinnebusCh, A.G. \& G.R. FinK: Repeated DNA sequences upstream from HISI also occur at several other co-regulated genes in Saccharomyces cerevisiae. J. Biol. Chem. 258, 5238-5247 (1983)

13. HONG, G.F.: A systematic DNA sequencing strategy. J. Mol. Biol. 158, 539-549 (1982)

14. Katsunuma, T.. S. ElSässer \& H. Holzer: Purification and some properties of threonine dehydratase from yeast. Eur. J. Biochem. 24, 83-87 (1971)

15. KYTE. J.\& R.F. DoolitTLE: A simple method for displaying the hydropathic character of a protein. J. Mol. Biol. 157, 105-132 (1982)

16. LuCChini, G., A.G. MinnebusCh, C. CHEN \& G.R. Fink: Positive regulatory interactions of the HIS4 gene of Saccharomyces cerevisiae. Molec. Cell Biol. 4, 1326-1333 (1984)
17. Martinez-Arias. A., H.J. Yost \& M.J. CasadaBAN: Role of upstream regulating element in leucine repression of the Saccharomyces cerevisiae leul2 gene. Nature 307, 740-742 (1984)

18. MESSING, J.: A multi-purpose cloning systembased on the single-stranded DNA bacteriophage M13. Recombinant DNA Tech. Bull., NIH publication No. 79-99, 2, 43-48 (1979)

19. MESSING.J. \& J. VIEIRA: A new pair of M1 3 vectors for selecting either strand of double-digest restriction fragments. Gene 19, 269-276 (1982)

20. NeUPeRT, W. \& G. SChatz: How proteins are transported into mitochondria. Trends in Biochem. Sci. 6, 1-4 (1981)

21. Petersen, J.G.l. S. Holmberg, T. NilssonTILLGREN\& M.C. KiELLAND-BRandT: Molecular cloning and characterization of the threonine deaminase (ILVI) gene of Saccharomyces cerevisiae. Carlsberg Res. Commun. 48, 149-159 (1983)

22. Pikielny, C.W., J.L. TeEm \& M. Rosbach: Evidence for the biochemical role of an internal sequence in yeast nuclear mRNA introns: Implications for UI RNA and metazoan mRNA splicing. Cell 34. 395-403 (1983)

23. Rasmussen, S.K., H.E. Hopp \& A. Brandt: Nucleotide sequences of $\mathrm{CDNA}$ clones for $\mathrm{B} 1$ hordein polypeptides. Carlsberg Res. Commun. 48, 187199 (1983)

24. Ryan. E.D. \& G.B. Kohlhaw: Subcellular localization of isoleucine-valine biosynthetic enzymes in yeast. J. Bacteriol. 120, 631-637 (1974)

25. SANGer, F., S. Nicklen \& A.R. Coulson: DNA sequencing with chain-terminating inhibitors. Proc. Nat. Acad. Sci. USA 74, 5463-5467 (1977)

26. Sentenac, A. \& B. Hall: Yeast nuclear RNA polymerases and their role in transcription. In: The Molecular Biology of the Yeast Saccharomyces cerevisiae. Metabolism and Gene Expression, J.N. Strathern, E.W. Jones \& J.R. Broach, eds. Cold Spring Harbor Laboratory 1982, pp. 561-606

27. StruHL, K.: The yeast his 3 promoter contains at least two distinct elements. Proc. Nat. Acad. Sci. USA 79, 7385-7389 (1982)

28. TINOCO, I., P.N. BoreR, B. Dengler, M.D. LeVINE, O.C. UHLENBECK, D.M. CROTHERS \& J. GRALLA: Improved estimation of secondary structure in ribonucleic acids. Nature New Biol. 246, 40-41 (1973)

29. Wettstein, D. von, C. Gjermansen, S. HolmBERG, M.C.KielLand-Brandt, T. NilsSON-TILL. GRen, M.B. Pedersen, J.G.L. Petersen \& P. SigsGAARD: Genetic engineering in the improvement of brewers yeast. MBAA Tech. Quarterly 21, 45-61 (1984)

30. YANG. R.C.-A., J. LIS \& R. WU: Elution of DNA 
from agarose gels after electrophoresis. In: Methods in Enzymology, R. Wu, ed. Academic Press, New York, vol. 68, pp. 179-182 (1979)

31. ZALKIN. H. \& C. YANOFSKY: Yeast gene TRP5: Structure, function, regulation. J. Biol. Chem. 257 , $1491-1500(1982)$

Accepted by: H. KLENOw, E. LUND and S.O. ANDERSEN
32. ZARET, K.S. \& F. Sherman: DNA sequence required for efficient transcription termination in yeast. Cell 28, 563-573 (1982) 